\title{
Simplified Brillouin sensor for structural health monitoring applications based on passive optical filtering
}

\author{
Haritz Iribas, Javier Urricelqui, Jon Mariñelarena, Mikel Sagues* and Alayn Loayssa \\ Universidad Pública de Navarra, Campus Arrosadia s/n, 31006 Pamplona, Spain
}

\begin{abstract}
We present a simplified configuration for distributed Brillouin optical time domain analysis sensors. The technique is based on passive optical filtering of the spectral components generated in an RF-pulse-modulated optical source. The aim of this configuration is to reduce the cost of the sensor by simplifying the generation of the optical waves involved in the sensing process. Proof-of-concept experiments demonstrate distributed temperature measurement with $1 \mathrm{~m}$ resolution over a $20 \mathrm{~km}$ sensing fiber.
\end{abstract}

Keywords: Brillouin distributed sensors, Brillouin optical time domain analysis (BOTDA).

\section{INTRODUCTION}

Over the last two decades, Brillouin fiber sensors have attracted great interest in the fiber-sensing community for their temperature and strain-monitoring capability. Among them, one of the most widely used approaches is the Brillouin optical time-domain analysis (BOTDA) technique, where a pump pulse interacts with a counter-propagating continuous wave $(\mathrm{CW})$ probe. BOTDA sensors can provide high precision distributed measurements of the strain and temperature profile over extremely large structures. This has made them very attractive for a wide set of industrial sectors, including oil and gas pipe monitoring, railway inspection and many other structural health monitoring applications. In this context, a number of research groups and sensing companies have focused their research on extending the capabilities of BOTDA sensors, improving their resolution ${ }^{1}$, extending their range ${ }^{2}$ or reducing their measurement time ${ }^{3}$.

However, in order to generate the optical waves involved in the technique, most BOTDA implementations use expensive components such as multiple electrooptic modulators, synthesized microwave generators or semiconductor optical amplifiers. As a consequence, this limits their real-world applications, which are usually bounded to industrial sectors where the cost of the sensor is not an issue. Therefore, a major line of research in these sensors is to simplify the technique in order to achieve cost-effective commercial systems that can compete with other less-costly technologies like, for instance, Raman sensors for temperature monitoring. Examples of contributions intended to reduce the cost of BOTDA sensors include the use of Brillouin fiber lasers to obtain the probe wave from the pump ${ }^{4}$, injection locking schemes to generate the pump and probe waves using inexpensive distributed feedback lasers ${ }^{5}$, the deployment of offset locking $^{6}$ or time-division pump-probe generation using direct modulation of a laser source ${ }^{7}$.

In this work, we present an alternative BOTDA sensor setup which is focused on reducing the potential cost of the sensor by obtaining all the optical waves involved in the technique by passive optical filtering of the spectral components of a pulsed-sideband modulated signal that is generated directly in the optical source. Besides, the technique is shown to be able to perform excellent distributed measurements with high accuracy and resolution, over several kilometers of fiber.

\section{FUNDAMENTALS}

The fundamentals of the proposed sensor setup are schematically depicted in Fig. 1. The first step is to generate an optical signal comprised of a continuous wave carrier and two pulsed modulated sidebands, which are directly generated in the optical source. Both the CW probe wave and the pulsed pump wave will be generated from this modulated signal: the optical carrier of the modulation will act as the $\mathrm{CW}$ probe wave, while one of the pulsed sidebands of the modulated signal will act as the pump wave. In order to do that, simple passive filtering is applied to the original signal, as described below. The RF-pulsed optical double-sideband (ODSB) signal is directed using a circulator to the upper

*mikel.sagues@ unavarra.es; phone 0034 948169729; fax 0034 948169720; unavarra.es 
branch of the setup. Here, the signal encounters a fiber Bragg grating (FBG) in transmission mode, tuned in such a way that the upper pulsed sideband of the modulation goes through it. Consequently, the other sideband and the optical carrier are reflected backwards, returning to the circulator, where they are directed into the fiber under test. Simultaneously, the pulsed sideband in the upper branch is injected from the other end of the fiber using a second circulator. In this way, both signals are counter-propagated in the fiber, where Brillouin interaction takes place. The modulation frequency is selected to be near the Brillouin frequency shift (BFS) of the fiber, so that the pump pulses can induce Brillouin amplification on the optical carrier. Then, the resultant probe wave is directed to a baseband photoreceiver and the detected electrical signal is captured in a digital oscilloscope. Finally, the measurement is repeated as the modulation frequency is swept, so that the Brillouin gain spectrum (BGS) of the fiber can be reconstructed.

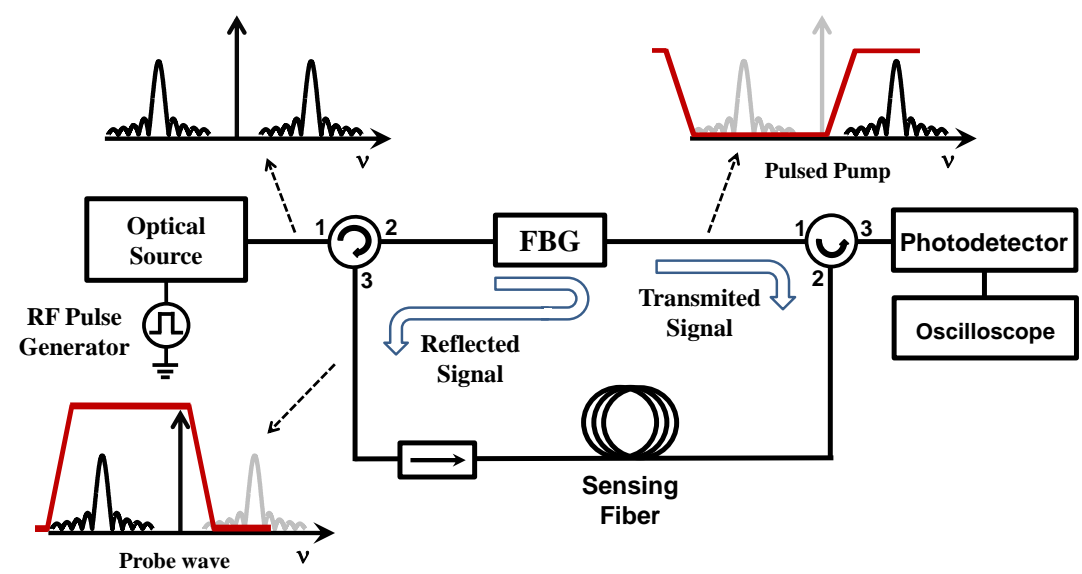

Figure 1. Schematic representation of the fundamentals of the technique. The spectra of the signals involved in the setup (in black) and the filter response (red) are sketched in the figure. The pulsed nature of the sidebands of the modulation is graphically represented by a sync spectrum.

The optical source can be implemented in several ways. For example, a distributed feedback laser (DFB) with a monolithically integrated electro-absorption modulator (EAM) could be deployed. Thanks to their possibility for monolithic integration with optoelectronic devices like DFBs and semiconductor optical amplifiers, EAMs have become a standard component for telecommunication applications. As a result, the optical source could be implemented by a single device, whose price in the market is comparable to that of a single laser source.

\section{EXPERIMENTS}

In order to study the capabilities of the proposed technique, the experimental setup which is depicted in Fig. 2 was assembled. The optical RF-pulsed modulation was implemented by external modulation of the output of a laser source, as no integrated DFB-EAM was available in the laboratory. Specifically, the output of a $1560.3 \mathrm{~nm}$ DFB laser source is directed to an EAM driven by a pulsed microwave signal. High extinction ratio RF pulses are generated deploying a microwave generator, followed by a microwave switch, which is driven by a pulse generator. In this way, the RF pulse shape is directly translated to the optical domain by the modulator. Besides, low cost commercial microwave switches are available with extremely high isolation and very fast responses (below $1 \mathrm{~ns}$ ). As a result, it is possible to obtain inexpensive and fast optical pulses with an extremely high extinction ratio $^{8}$.

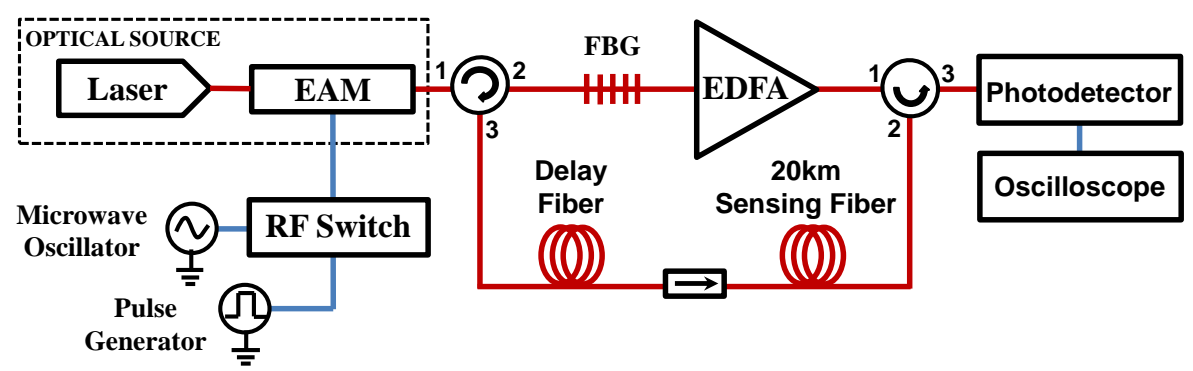

Figure 2. Experimental setup for the low cost BOTDA sensor based on pulsed ODSB modulation and passive optical filtering. 
The resulting modulated signal is directed to the upper branch of the setup, where it is filtered employing a narrowband FBG. A gain-based BOTDA sensor was arranged by tuning the FBG so that the pulsed upper sideband of the modulation is transmitted, while the other sideband and the CW optical carrier are reflected. Nevertheless, the technique can also be applied to loss-based BOTDA sensors, by simply selecting the lower pulsed sideband of the modulation. Then, the pulsed pump wave at the output of the FBG is amplified with an erbium-doped fiber amplifier to a close level before modulation instability appears ${ }^{9}$. Before being launched into the sensing fiber, the polarization of the pulsed pump is randomized with a polarization scrambler, so as to reduce polarization-mismatching-induced fluctuations on the signal. Finally, the probe signal is detected in a $125 \mathrm{MHz}$ photoreceiver and the resultant BOTDA signal is captured in a digital oscilloscope.

Notice that, as a result of generating an optical RF-pulsed modulated signal with a double-sideband modulator, two pulsed sidebands are co-propagating with the probe wave in the fiber. The pulsed lower sideband of the modulation (which is reflected by the FBG), and the remaining upper pulse (which is not perfectly filtered by the FBG). As a consequence, when they reach the oscilloscope, these unwanted pulses will corrupt the measurement in the middle section of the fiber. This limitation can be overcome in a number of ways, as deploying a second FBG in the lower branch so that these pulses are further attenuated; or deploying an optical single sideband modulator in order to suppress the lower sideband of the modulation. In our experimental setup we insert a dummy length of fiber before the isolator in the lower branch of the setup. This acts as a delay line, so that we can make the unwanted pulses reach the receiver once the whole BOTDA trace corresponding to the sensing fiber has been captured.

In order to analyze the performance of the system, distributed temperature measurements were performed over a $20 \mathrm{~km}$ length of standard single-mode fiber. The pulse duration was set to $10 \mathrm{~ns}$, corresponding to approximately $1 \mathrm{~m}$ resolution, and 4096 averages were made in the oscilloscope. The final $10 \mathrm{~m}$ of the fiber were placed loose in a climatic chamber, while the rest was held at room temperature in a reel. The modulation frequency was swept at $2 \mathrm{MHz}$ steps.

The temperature of the climatic chamber was modified in order to evaluate the accuracy of the technique for temperature measurements. The measured spectrum at the heated section of the fiber is depicted in Fig. 3(a). The BFSs corresponding to the amplitude spectra as the temperature is risen were calculated by performing a mathematical fit considering a Lorentzian-Gaussian profile. This is depicted in Fig. 3(b), where the linear regression performed to the BFS data obtained from the spectra shows a clear linear relation with temperature. Besides, there is a good agreement between the changes of temperature at the climatic chamber $\left(10^{\circ} \mathrm{C}\right.$ per measurement $)$ and the frequency shift of the spectra.

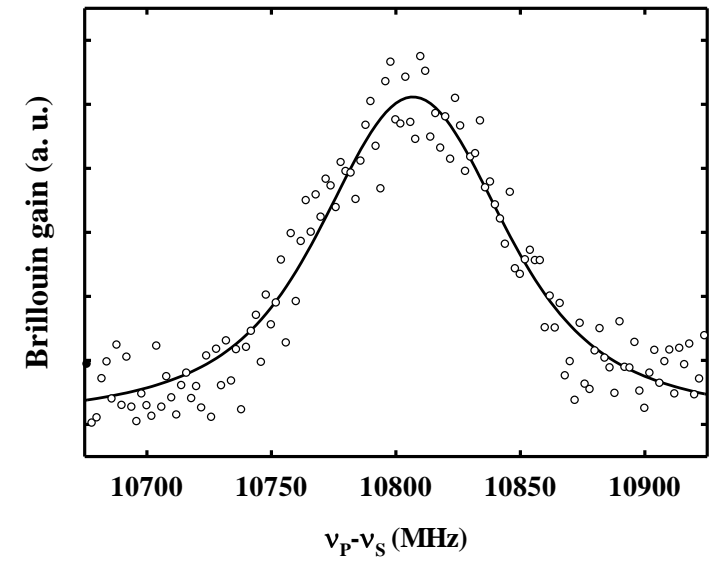

(a)

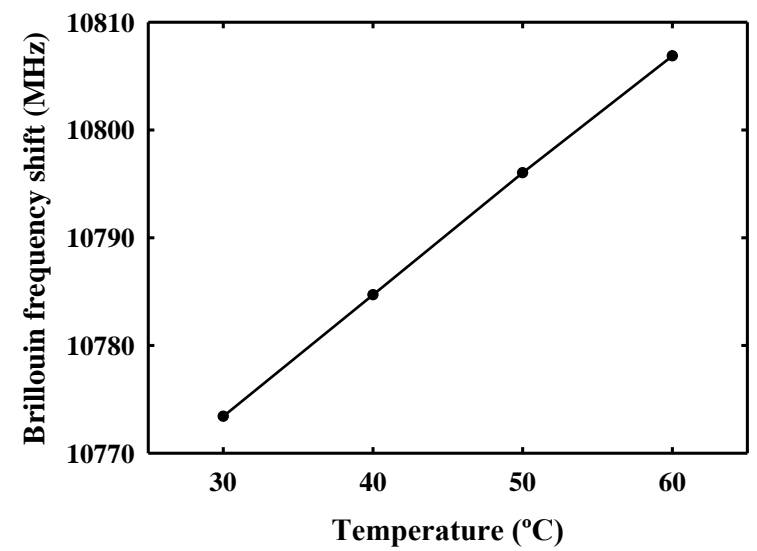

(b)

Figure 3. (a) Brillouin gain spectrum measured (symbols) at the heated section located at the end of the fiber. The mathematical FIT is also shown in the figure (solid line). (b) Calculated BFS as a function of temperature in the climatic chamber (symbols) and linear regression (solid line) as the temperature is risen in $10^{\circ} \mathrm{C}$ steps.

Finally, we analyze the performance of the sensor for distributed measurements. Fig. 4(a) depicts the evolution of the measured Brillouin frequency shift at the end of the fiber, when different temperatures are applied to the heated section of the fiber. The 10-m-section corresponding to the heated section are clearly visible and a fast transition between the 
heated section and the rest of the fiber is also observed. The spatial resolution was confirmed to be $1 \mathrm{~m}$ by measuring the rise time between two adjacent sections of the fiber at different temperatures.

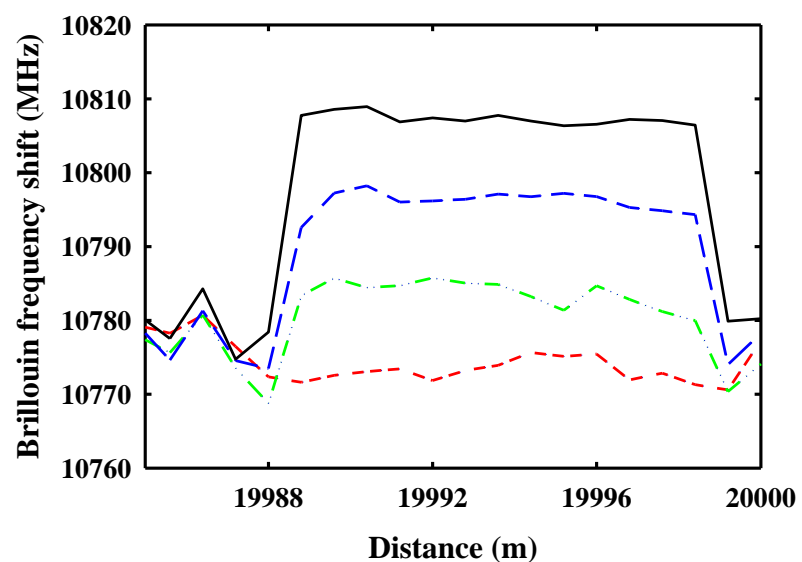

Figure 4. Calculated BFS as a function of distance at the final locations of the fiber, as the temperature is risen in the climatic chamber in $10^{\circ} \mathrm{C}$ steps.

\section{CONCLUSIONS}

A simplified configuration for Brillouin distributed sensors has been proposed and demonstrated. The sensor relies on passive optical filtering of the spectral components of an RF-pulsed modulated signal. This reduces the number and price of the components required to implement the sensor, reducing its final cost. The capabilities of the technique have been demonstrated, performing distributed temperature measurements over a $20 \mathrm{~km}$ length of fiber with high precision and resolution. Further work should focus on improving the performance of the sensor, as well as finding other simplified configurations, so that BOTDA sensors can reach applications and industries from which they are now excluded.

\section{ACKNOWLEDGEMENTS}

The authors wish to acknowledge the financial support from the Spanish Ministerio de Ciencia e Innovación through the project TEC2013-47264-C2-2-R, FEDER funds and Universidad Pública de Navarra.

\section{REFERENCES}

[1] Li, W., Bao, X., Li, Y. and Chen, L., "Differential pulse-width pair BOTDA for high spatial resolution sensing," Opt. Express 16(26), 21616-21625 (2008).

[2] Angulo Vinuesa, X., Martin, S., Corredera, P. and Gonzalez Herraez, M., "Raman assisted Brillouin optical time domain analysis with sub meter resolution over 100 km," Opt. Express 20(11), 12147-12154 (2012).

[3] Urricelqui, J., Zornoza, A., Sagues, M. and Loayssa, A., "Dynamic BOTDA measurements based on Brillouin phase-shift and RF demodulation,” Opt. Express 20(24), 26942-26949 (2012).

[4] Lecoeuche, V., Webb, D. J., Pannell, C. N. and Jackson, D. A., "Brillouin based distributed fibre sensor incorporating a mode-locked Brillouin fibre ring laser," Opt. Commun. 152(4-6), 263-8 (1998).

[5] Thévenaz, L., Le Floch, S., Alasia, D. and Troger, J., "Novel schemes for optical signal generation using laser injection locking with application to Brillouin sensing," Meas. Sci. Technol. 15, 1519-24 (2004).

[6] Li, Y., Bao, X., Ravet, F. and Ponomarev, E. "Distributed Brillouin sensor system based on offset locking of two distributed feedback lasers," Appl. Opt. 47(2), 99-102 (2008).

[7] Song, K. and Yang, S., "Simplified Brillouin optical time-domain sensor based on direct modulation of a laser diode," Opt. Express 18(23), 24012-24018 (2010).

[8] Zornoza, A., Olier, D., Sagues M. and Loayssa, A., "Brillouin distributed sensor using RF shaping of pump pulses," Meas. Sci. Technol. 21, 094021 (2010).

[9] Alahbabi, M. N., Cho, Y. T., Newson, T. P., Wait, P. C., Hartog, A, H., "Influence of modulation instability on distributed optical fibre sensors based on spontaneous Brillouin scattering," JOSA B 21(6), 1156-1160 (2004). 